\title{
A PESQUISA EM ENFERMAGEM NO BRASIL E SUA POSIÇÃO EM AGÊNCIA FEDERAL DE FOMENTO
}

leda de Alencar Barreira*

\begin{abstract}
O trabalho apresenta uma retrospectiva histórica das atividades de pesquisa em enfermagem no Brasil, com destaque para a sistematização, as tendências e a difusão dessas pesquisas, e em especial para o fomento das mesmas pelo Conselho Nacional de Desenvolvimento Científico e Tecnológico, apresentando as origens e a estrutura deste órgão. Ao discutir a inserção da enfermagem nesta agência de fomento, é relatada a situação contraditória entre a proposta oficial para a formação do enfermeiro e a orientação oferecida pelo Conselho Nacional de Desenvolvimento Científico e Tecnológico para contemplar a área de enfermagem. O estudo traz ainda uma reflexão acerca da importância da definição em linhas de pesquisa e enfermagem visando às exigências atuais do processo de produção de conhecimento.
\end{abstract}

\section{Considerações iniciais}

Com atividade sistemática e regular, a pesquisa em enfermagem no Brasil tem menos de 20 anos, ocorrendo de modo predominante sob a forma de uma prática acadêmica, decorrente de estratégias governamentais condizentes com as ideologias do Estado empresarial e da Segurança Nacional, adotadas pelo regime militar, que se instalou no país em 1964. No que se refere ao ensino superior, tais estratégias foram veiculadas pela Reforma Universitária (Lei $n^{\circ}$ 5540/68) que, ao redefinir a carreira do magistério a partir da qualificação dos docentes em nível de pós-graduação, fixando o princípio da unidade-pesquisa, propiciou a criação dos primeiros cursos de mestrado em enfermagem. Assim é que, entre 1972 a 1978 foram criados nove desses cursos que deram novo impulso à pesquisa nessa área.

Como desde a implantação do sistema "nightingale" entre nós, a enfermagem brasileira de então continuava sob forte influência dos Estados Unidos da América, e deste modo nossa pós-graduação, não obstante a importância da contribuição de algumas educadoras brasileiras, nasceu sob o signo das pesquisas e das teorias das enfermeiras norte-americanas, muitas das quais tentavam então afirmar a enfermagem como ciência, ou pelo menos como ciência em construção em uma perspectiva positivista. Desde seu início, a pesquisa em enfermagem surge trespassada pelas incertezas devidas à sua dupla filiação: à biologia e à medicina, por um lado e às ciências sociais e à educação por outro lado, dentro de um marco conceitual funcionalista.

Foi somente com o advento da "abertura política" que caracteriza os anos 80 na América Latina, que veio ocorrer entre nós uma discussão mais ampla das diversas tendências de pensamento. Aliás, é no início da década de 80 que a nossa produção cientifica de enfermagem torna-se objeto de estudo de várias enfermeiras pesquisadoras, que a analisaram sob diferentes perspectivas. Entre outros aspectos destacados nessas análises, ressalta uma hegemonia geográfica e institucional na

*Professora Titular da Escola de Enfermagem de Anna Nery, da Universidade Federal do Rio de Janeiro e Consultora de Enfermagem junto ao Conselho Nacional de Pesquisa e Desenvolvimento Científico e Tecnológico (CNPq), para o período de 1990 a 1992. 
produção científica de enfermagem, diretamente relacionada a universidades das regiões sudeste e sul, que desenvolvem cursos de pós-graduação em enfermagem, e indiretamente à concentração de rendas nessas regiões, relativamente ao país.

A contribuição dessa produção científica para o encaminhamento das questões em enfermagem tem sido bastante questionada. É baixa a difusão dos trabalhos produzidos, devido a problemas editoriais de distribuição e de consumo dessa literatura. Assim, a produção de pesquisas em enfermagem tem sido insuficientemente aproveitada, mesmo no ensino de pósgraduação e no seu aproveitamento nos cursos de graduação e é mais problemático. Na área de serviço, seu desconhecimento é maior, até mesmo pelo fato de que a maioria desses trabalhos é de autoria de professoras de enfermagem. O fenômeno da formação de uma elite intelectual no âmbito da pós-graduação mais se acentua com a criação dos cursos de doutorado, a partir de 1980, em contraste com a qualidade da assistência de enfermagem em geral, que por vezes apresenta padrões inaceitáveis.

Outro lado da questão é que, centro de interesse de muitos desses trabalhos era os aspectos internos da prática profissional, enquanto prática técnica, ao passo que as raízes dos problemas que se manifestam no trabalho cotidiano de enfermagem muitas vezes encontram-se externamente a ela. Diante dessa compreensão é que nos últimos anos começaram a aumentar o número de estudos de caráter histórico-estrutural, que buscam explicações para a problemática da enfermagem e encaminhamentos mais realísticos para suas questões. Também se vem incrementando o número de pesquisas de abordagem fenomenológica, que buscam descrever e compreender em profundidade as experiências vividas no âmbito da enfermagem, segundo a perspectiva pessoal e o significado a elas atribuído por quem as viveu.

Mas, o aumento progressivo do número de trabalhos científicos elaborados não se fez acompanhar por um aumento correspondente de espaços para publicação. Neste sentido, vale destacar a participação da Associação Brasileira de enfermagem (ABEn), criada em 1926, ainda nos primórdios da profissão no Brasil, ao editar a Revista Brasileira da Enfermagem, fundada em 1936 e, desde meados da década de 70, os anais de seus congressos anuais e dos seminários bienais de pesquisa, promovidos pelo seu Centro de Pesquisas. Também algumas escolas e cursos de enfermagem, bem como algumas seções estaduais da ABEn criaram revistas que vêm contribuindo para a publicação da produção científica das enfermeiras. O primeiro trabalho com grandes números para o diagnóstico da situação da enfermagem no Brasil foi realizado pela ABEn, de 1956 a 1958, com apoio da Organização Mundial de Saúde e da Fundação Rockfeller. O segundo trabalho desse teor foi realizado por iniciativa do Conselho Federal de Enfermagem, em convênio com ABEn e o Instituto Nacional de Assistência Médica da Previdência Social, com apoio da Organização Panamericana de Saúde e do Conselho Nacional de Desenvolvimento Científico e Tecnológico (CNPq) e publicado em 1986.

\section{O Conselho Nacional de Desenvolvimento Científico e Tecnológico (CNPq) - origens, estrutura atual e inserção da enfermagem.}

O Conselho Nacional de Pesquisas foi fundado em 1951, como órgão diretamente subordinado à presidência da República. A criação do CNPq, fundado pelo cientista Álvaro Alberto, almirante, pesquisador da energia atômica e representante do Brasil na $1^{\text {a }}$ Reunião da Comissão de Energia Atômica da ONU (1946), decorreu da necessidade de evitar a desapropriação das nossas reservas de material radioativo. Em 1964, o Conselho teve ampliado sua área de competência, que passou a abranger também a formulação da política científica e tecnológica do país, bem como a coordenação dessas atividades; mas as atividades de execução de pesquisa no campo da energia atômica deixaram de ser sua área de competência. A partir de 1972 começaram os Planos Básicos de Desenvolvimento Científico e Tecnológico/PBDCTs, integrados aos Planos Nacionais de Desenvolvimento/PNDs. Em 1974, o CNPq transformou-se em fundação de direito privado, com a denominação Conselho Nacional de Desenvolvimento Científico 
e Tecnológico, com a mesma sigla, vinculada à Secretaria de Planejamento da Presidência da Republica/Seplan. Posteriormente o CNPq ficou ligado diretamente à Presidência da Republica, e cujo objetivo-síntese era a modernização científica e tecnologia do país. Sua missão era definida como a de "promover e apoiar o desenvolvimento científico e tecnológico nacional", sendo o CNPq o órgão executar da política nacional de ciência e tecnologia (C\&T). A área de influência do CNPq abrange todas as áreas do conhecimento e todo o território nacional. $\mathrm{Na}$ década de 80 o orçamento de bolsas do CNPq cresceu a uma taxa média anual de $300 \%$, elevando o número de bolsas de 7200, em 1980, para 44 mil em 1990 (CNPq,1991). A partir dos anos 90, no bojo de uma crise nacional de caráter econômico e político, o CNPq entrou em um processo de desgaste, devido a crescentes restrições orçamentárias, que passaram comprometer o financiamento de bolsas e auxílios e, ao mesmo tempo, a situação salarial dos quadros de pesquisadores, técnicos e funcionários do próprio CNPq, bem como dos dez institutos de pesquisa que integram sua estrutura.

O CNPq tem três Superintendências, cada qual abrangendo um número variável de coordenadorias:

01) Superintendência de Engenharias, Ciências Exatas e da Terra;

02) Superintendência de Ciências Humanas e Sociais;

03) Superintendência de Ciências da Vida, onde se inclui a Coordenadoria de Ciências da Saúde/COCS.

Somente a partir de 1986 passou a existir no CNPq uma representante de enfermagem como membro do comitê assessor, bem como uma enfermeira como técnico de desenvolvimento científico, ambas atuando junto á COCS. Esta coordenadoria atualmente compreende três Comitês Assessores (CAs), entre os quais o CA Saúde Complementar, constituído pelas áreas de educação física, enfermagem, fisioterapia e terapia ocupacional, fonoaudiologia, e odontologia.

Assim é que a enfermagem no CNPq encontra-se inserida em uma coordenação de orientação nitidamente biológica, enquanto que a proposta oficial dos cursos de graduação vem sendo a de preparar profissionais para prestar uma assistência global a pessoas ou grupos e, nos serviços de saúde, as atividades de enfermeira são principalmente de cunho administrativo, isto é, da organização do processo de trabalho de enfermagem", que apelam para uma abordagem das ciências sociais.

Outro tipo de desvantagem com que se depara a enfermagem no CNPq é o nivelamento de todas as áreas por critérios técnico-normativos uniformes, sem considerar o grau de maturação da área. Por exemplo, 1990 todos os detentores de uma bolsa de pesquisador que não possuíssem o título de doutor ( ou equivalente) foram excluídos do sistema, o que fez com que um grande número de enfermeiras pesquisadoras, perdessem suas bolsas. Assim, como se pode observar na Tabela 1, em 1991, encontravam-se no sistema apenas 49 pesquisadoras, distribuídas entre os níveis I ( $a, b$ ou c) e II ( $a, b$ ou c), notando-se uma concentração de quase a metade dos pesquisadores na categoria II c (doutores há menos de quatro anos); se considerarmos o total do nível II, atingiremos mais $3 / 4$ das pesquisadoras.

TABELA 1: BOLSAS DE PESQUISA NA ÁREA DE ENFERMAGEM, SEGUNDO CATEGORIAS E SUB-CATEGORIAS. CNPq, 1991

\begin{tabular}{|c|c|c|c|c|c|c||}
\cline { 2 - 7 } \multicolumn{1}{c|}{} & \multicolumn{2}{c|}{ I } & \multicolumn{2}{c|}{ II } & \multicolumn{2}{c|}{ TOTAL } \\
\cline { 2 - 7 } \multicolumn{1}{c|}{} & $\mathbf{N .}^{\mathbf{0}}$ & $\mathbf{\%}$ & $\mathbf{N .}^{{ }^{\circ}}$ & $\mathbf{\%}$ & $\mathbf{N .}^{{ }^{\circ}}$ & $\mathbf{\%}$ \\
\hline A & 1 & 2,0 & 8 & 16,3 & 9 & 18,4 \\
\hline B & 3 & 6,1 & 6 & 12,2 & 9 & 18,4 \\
\hline C & 7 & 14,3 & 24 & 48,8 & 31 & 63,3 \\
\hline TOTAL & $\mathbf{1 1}$ & $\mathbf{2 2 , 4}$ & $\mathbf{3 8}$ & $\mathbf{7 7 , 5}$ & $\mathbf{4 9}$ & $\mathbf{1 0 0 , 0}$ \\
\hline
\end{tabular}

Fonte: Documentação CA/SC - 6/91 e 10/91 
A passagem do nível II para o nível I exige uma produção científica regular de qualidade e independente, orientação de novos pesquisadores, autonomia e originalidade. A avaliação dos pesquisadores se faz em grande parte mediante sua produção científica, inclusive para a progressão de sua classificação no CNPq, segundo critérios de indexação, peso do Conselho editorial, utilização de avaliadores externos à revista, percentual de trabalhos de pesquisa por número da revista, qualidade gráfica, o que coloca as pesquisadoras em grandes dificuldades.

Também devido ao reduzido número de enfermeiras com o título de doutor, a disponibilidade de bolsas que exigem esse título (recém-doutor, pós-doutorado, pesquisador visitante, pesquisador associado) vem sendo maior do que a procura. Ainda mais, como há uma demanda reprimida, a média de idade dos candidatos à bolsa de doutorado é mais elevada do que nas áreas em que a pesquisa já se encontra consolidada; no entanto, a idade limite considerada adequada a um solicitante deste tipo de bolsa é de trinta e cinco anos.

\section{Projetos de pesquisa em enfermagem apoiados pelo CNPq}

Os dados da tabela 2, a seguir, permitem comparar os projetos apresentados ao CNPq e os trabalhos apresentados no $6^{\circ}$ Seminário Nacional de Pesquisa em Enfermagem, promovido pelo Centro de Pesquisas da $A B E n$, no que se refere à sua distribuição entre áreas temáticas, conforme classificação do $2^{\circ}$ Seminário Nacional de Pesquisa (1981):

\section{Área I - PROFISSIONAL}

1.1 - A enfermagem como prática social.

1.2 - Formação e utilização de recursos humanos em enfermagem

\section{Área II - ASSISTENCIAL}

2.1 - Fundamentação da assistência, tecnologia e instrumentação

2.2 - Autocuidado à saúde

2.3 - Riscos da assistência de enfermagem

2.4 - Determinantes do processo saúde-doença

\section{Área III - INSTITUCIONAL}

3.1 - Modelo de assistência de enfermagem

3.2 - Oferta, acessibilidade a utilização de serviços de saúde

3.3 - Sistema de informação em enfermagem

3.4 - Incorporação de conhecimento à prática profissional

3.5 - Dinâmica das organizações 
Tabela 2: Classificação da produção científica apresentada no $6^{\circ}$ SEMPE/1001 e projetos apoiados pelo CNPq em 1991, conforme classificação proposta pelo $2^{\circ}$ SEMPE (1981)

\begin{tabular}{|c|c|c|c|c|}
\hline $\begin{array}{c}\text { Eventos } \\
\text { Áreas }\end{array}$ & $\begin{array}{c}6^{\circ} \text { SENPE } \\
\text { N. }\end{array}$ & $\begin{array}{c}\text { (Rio, 1991) } \\
\%\end{array}$ & $\begin{array}{c}\text { PROJETOS } \\
\text { N. }^{\circ}\end{array}$ & $\begin{array}{c}\text { (CNPq, 1991) } \\
\%\end{array}$ \\
\hline Profissional & 28 & 41,8 & 13 & 20,3 \\
\hline 1.1 & 18 & 26,8 & 4 & 6,2 \\
\hline 1.2 & 10 & 15,0 & 9 & 14,1 \\
\hline Assistencial & 22 & 32,8 & 37 & 57,9 \\
\hline 2.1 & 5 & 7,5 & 6 & 9,4 \\
\hline 2.2 & 6 & 8,9 & 9 & 14,1 \\
\hline 2.3 & - & - & 3 & 4,7 \\
\hline 2.4 & 11 & 16,4 & 19 & 29,8 \\
\hline Institucional & 17 & 25,4 & - & - \\
\hline 3.1 & 2 & 3,0 & 5 & 7,8 \\
\hline 3.2 & 3 & 4,5 & 2 & 3,1 \\
\hline 3.3 & 3 & 4,5 & 3 & 4,7 \\
\hline 3.4 & 9 & 13,4 & 2 & 3,1 \\
\hline 3.5 & - & - & 2 & 3,1 \\
\hline TOTAL & 67 & 100,0 & 64 & 100,0 \\
\hline
\end{tabular}

Fontes: Comissão de Temas $6^{\circ}$ SENPE e CNPq, CA/SC 6/91 e 10/91

Observa-se uma coincidência quantitativa entre os dois conjuntos de trabalhos, ao lado de uma grande divergência temática, pois enquanto que no $6^{\circ}$ SEMPE ocorre um predomínio da área profissional, decorrente de um grande número de trabalhos sobre e enfermagem como prática social, no que se refere ao CNPq este predomínio ocorre na área assistencial, que supera as das outras áreas reunidas. Nota-se ainda a concentração dos trabalhos da área assistencial na linha "determinantes do processo saúde-doença", em ambos os conjuntos analisados.

\section{Linhas de pesquisa em enfermagem}

A importância de se definir linhas de pesquisa em enfermagem prende-se às exigências atuais do processo de produção do conhecimento, que se tornou um empreendimento coletivo, realizado de modo progressivo e contínuo ao longo do tempo. No entanto uma linha de pesquisa passa a ter existência como resultado da produção mesma de investigações, que, no entanto deve atender a certas condições. Por isso, pode não ser tarefa fácil identificar o nascimento de uma linha de pesquisa. E tanto assim que, sendo um dos objetivos da Reunião Nacional de Coordenadores de Cursos de PósGradução em enfermagem (1991) o de inventariar as linhas de pesquisa existentes 
e em construção nas instituições de ensino de enfermagem, diante das dificuldades apresentadas por tal tarefa, foram elaboradas critérios para a avaliação do grau de consolidação de uma linha de pesquisa, segundo sua persistência temporal, produção científica e maturidade das equipes, considerando-se três níveis:

\section{Nível 1: Linha de Pesquisa Consolidada}

- Trabalho coletivo com duração de cerca de cinco anos, que envolve pessoas em vários níveis de formação e experiência em pesquisa, receptivo a novos pesquisadores, no qual a produção já existente apresenta um forte nexo entre os diferentes projetos, que se encontram voltados para uma questão norteadora, e que favoreça ainda o intercâmbio institucional ou interdisciplinar.

\section{Nível 2: Linha de Pesquisa Emergente}

- Trabalho coletivo realizado em torno de uma questão norteadora, com alguma produção já existente e projetos em andamento, com tendências à ampliação e consolidação.

\section{Nível 3: Produção Científica Isolada}

- Pesquisas individuais e/ou grupais, sem necessariamente estabelecerem vínculos de internacionalidade em torno de uma única questão norteadora, mas que representam parcela importante de produção científica da unidade.

O estabelecimento desses critérios veio a possibilitar às instituições de ensino de enfermagem melhor avaliarem o grau de institucionalização da pesquisa produzida por professores e alunos, e ainda mais, incentivar seu agrupamento em núcleos de pesquisa, que possam integrar, a cada momento, novos pesquisadores, com experiência variável. O número, variedade e distribuição de núcleos ativos de pesquisa em enfermagem expressam a situação da pesquisa na área e dão forte respaldo aos pleitos de seus pesquisadores junto às agências de financiamento.

This study presents a historical retrospective of research activities in nursing in Brazil, with emphasis on systematization, tendencies and diffusion of this studies, especially for the support of these by the National Council of Scientific and Technological Development. The origins and actual structure of this agency is also presented. In discussing the Insertion of nursing in this agency of financial support, the contradictory situation between the official proposal for the training of a nurse and the orientation offered by the National Council of Scientific and Technological Development is reported, This study also offers reflection about the importance of the definition of research lines nursing, in view of the demands of the process of knowledge production.

El trabajo presenta una retrospectiva histórica de las actividades de Investigación en Enfermería en Brasil, con evidencia para la sistematización, las tendencias y la difusión de esas investigaciones, y en especial para el fomento de las mismas por el CNPq (Consejo Nacional de Desenvolvimiento Científico y Tecnológico) presentando también las orígenes y la estructura actual de este órgano. Al discutir la inserción de la Enfermería en esta Agencia de fomento, es relatada la situación contradictoria entre la propuesta oficial para la formación del Enfermero y la orientación ofrecida por el CNPq para contemplar el área de Enfermería. El estudio trae todavía una reflexión acerca de la importancia de la definición de líneas de investigación en Enfermería, objetivando las exigencias actudes del proceso de producción del conocimiento.

\section{REFERÊNCIAS BLIBLIOGRÁFICAS}

01. BARREIRA, I. de A. - Informativo Enfermagem \& Conselho Nacional de Desenvolvimento Científico e Tecnológico. Rio de Janeiro, 1991. (Mimeografado)

02. BRASIL. Presidência da República. Plano Plurianual de Diretrizes e Metas 1991/1995, p. 6. 
03. BRASIL. Presidência da República. Conselho Nacional de Desenvolvimento Científico e Tecnológico. Agenda CNPq, 13 (60), 1991.

04. CASTRO, I. B.; ALMEIDA, M. C. P. La investigación en enfermería como instrumento de cambio en la práctica y en la formación de recursos humanos. Educ. Méd. Salud, v. 19, n. 3, Washington, 1985.

05. CASTRO, I. B.; TYRREL, M. A. R. Fontes de dados para o ensino e a pesquisa em enfermagem. Rio de Janeiro, maio, 1991. (Mimeografado).

06. CASTRO, I. B. Programa Básico de Enfermagem na Coordenação de Ciências da Saúde. Conselho Nacional de Desenvolvimento Científico e Tecnológico. Brasília, 1991. (Mimeografado).

07. WRIGHT, M. da G. M. Programa Básico de Enfermagem na Coordenação de Ciências da Saúde. Conselho Nacional de Desenvolvimento Científico e Tecnológico. Brasília, 1988. (Mimeografado).

08. Reunião Nacional de Coordenadores de Cursos de Pós-Graduação "Stricto Sensu" da Área de Enfermagem e da Comissão "ad-hoc". Coordenação de Aperfeiçoamento de Pessoal de Nível Superior/Conselho Nacional de desenvolvimento Científico e Tecnológico/Associação Brasileira de Enfermagem. Brasília, novembro de 1991. (Mimeografado). 